creased virulence. But in as much as in a number of experiments such an excluding action of a first inoculation was not noticeable, we are not yet ready to state positively that such an immunizing effect of a growing tumor exists. We are however inclined to believe that a number of variable factors (especially a variable energy of growth of the first and second tumors) are present and that such factors complicate the results. In further experiments we shall endeavor to analyze such variable factors.

I5 (540)

\title{
Supplementary report on attempts to immunize against tuberculosis.
}

\section{By J. P. Atkinson and C. B. FitzPatrick.}

[From the Department of Health, City of New York.]

We have sought to employ the resistance acquired by or embodied in the serum of the healthy animal in its life struggle against a deadly infection with tuberculosis.

The depression reaction produced by injections of tuberculous serum into animals sensitised with tuberculin, a description of which we have already published has led us to this investigation.

After employing a number of methods for utilizing the serum of healthy animals which had been infected with tuberculosis, we are duplicating one which seems to have given promising results.

Method I-Begun December 2, I909. The result of this experiment was reported at the meeting of the American Association for the Advancement of Science, December 27, 1909.

One rabbit was given a sensitising dose of I c.c. tuberculin subcutaneously and beginning two days later this rabbit received the following:

$\begin{array}{cr}\text { Dec. } & 4, \\ \text { “ } & 6, \\ “ & 8, \\ \text { “ } & 10, \\ \text { “ } & 14, \\ \text { “ } & 16, \\ \text { “ } & 18,\end{array}$

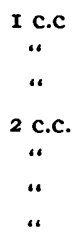

Tuberculous rabbit serum.

A rest of ten days was given the experimental rabbit which was 
then given a fatal dose of a culture of bovine tuberculosis intravenously. Two control rabbits were given an equal amount of the same culture. The controls lived 30 days during which time they lost weight and presented a typical tubercular appearance. The autopsy showed marked and characteristic lesions of tuberculosis.

The experimental rabbit lived 39 days and kept in good condition up to one week before its death and then lost weight rapidly. The autopsy showed the same marked and characteristic tubercular lesions as the controls.

The fact of the long-continued good physical condition of the experimental rabbit encouraged us to repeat this experiment with some changes.

January 8 , I9Io. Two rabbits were sensitised with $1 / 4$ c.c. each of crude tuberculin which was followed on the twelfth inst., by injections of 3 c.c. each of tuberculous rabbit serum. This procedure was repeated three times and the serum alone given six times.

The following is the table of injections, all subcutaneous.

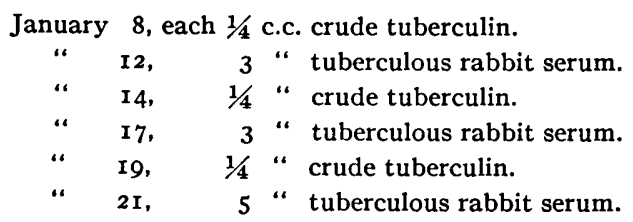

One rabbit died on January $2 \mathrm{I}$. The other one received on January 24, 1/4 c.c. crude tuberculin.

“ 26, 5 " tuberculous rabbit serum.

“ $28,21 / 2$ "

“30,

February 3, $21 / 2$

“ 5 ,

3 c.c. tuberculous rabbit serum.

“ 7 ,

$\begin{array}{lllll}5 & \text { “ } & \text { " } & \text { " } & \\ 5 & \text { “ } & \text { " } & \end{array}$

A rest of I I days was given the surviving rabbit, and then on February 18, 1910, it was given a fatal dose of bovine tuberculosis intravenously. Two control rabbits were given the same amount of the bovine tuberculosis culture.

One control died April 5, the second control died July II; both these rabbits showed marked and characteristic tubercular lesions. The experimental rabbit died July $\mathrm{I} 7$, from pneumonia. 
The autopsy showed that the lungs, liver, kidneys, suprarenal capsule, spleen and glands were free from tubercular infection.

Both controls lost weight consistently until death.

The experimental rabbit held its weight up to a week before its death when it lost weight very rapidly.

This rabbit appears to have been successfully protected by injections of tuberculin and tuberculous rabbit serum from a fatal dose of a culture of bovine tuberculosis. We are now duplicating this experiment with two experimental rabbits and three controls.

On June 25, 1910, two variations of the previous methods were used. Two rabbits were used for each experiment. Variation $\mathrm{I}$ consisted in alternate injections of crude tuberculin and tuberculous rabbit serum as follows:

June 25 , each rabbit $1 / 4$ c.c. tuberculin.

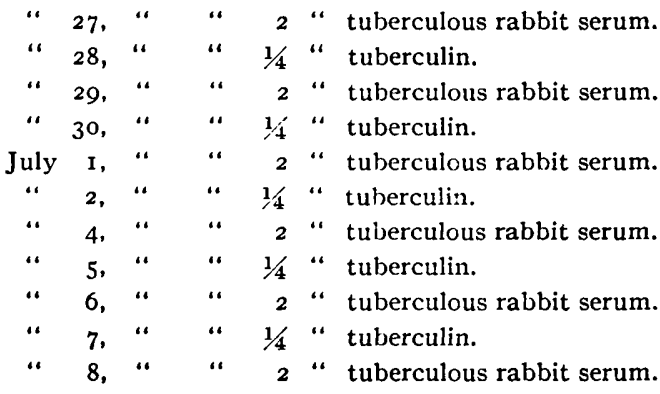

Variation 2 consisted in alternate injections of tuberculous rabbit serum and that portion of the tubercle bacillus split by alcoholic potash which is insoluble in the alcohol. Vaughan claims that this portion of the tubercle bacillus sensitises but does not poison. This is true of the preparation we used as will be described further on.

The following table gives the injections:

June 25 , each rabbit $\mathrm{x} / \mathrm{r} 0 \mathrm{gm}$. insoluble residue.

" 27, , " 22 c.c. tuberculous rabbit serum.

“ 28, “ " " I/ro $\mathrm{gm}$. insoluble residue.

“ 29, “ “ $“ 2$ c.c. tuberculous rabbit serum.

" 30, " " $\quad$ I/10 $\mathrm{gm}$. insoluble residue.

July I, “ “ $\quad 2 \quad 2 \quad$ c.c. tuberculous rabbit serum.

“ 2 , " " $\quad 1 / 10 \mathrm{gm}$. insoluble residue.

“ 4 , “ “ 2 c.c. tuberculous rabbit serum.

“ 5, " " " $\quad$ I/ro gm. insoluble residue.

“ 6, " $\quad$ " $\quad 2 \quad 2$ c.c. tuberculous rabbit serum.

“ 7, " " $1 / 10 \mathrm{gm}$. insoluble residue.

“ $8, “ \quad$ “ 2 c.c. tuberculous rabbit serum. 
(One of these rabbits was bitten and died from a resulting infection, August I.)

These four rabbits with three controls were each given an intravenous injection of a lethal dose of bovine tuberculosis on July 22, 1910, after an interval of 12 days since the last injection of tuberculous rabbit serum.

These rabbits died and showed marked and characteristic lesions upon autopsy except the rabbit which died on August I, as the result of a bite and one that died August 22, and was not autopsied. This animal, however, showed physical signs of tuberculosis before death.

The following table shows the time of death of the experimental rabbits and the controls.

$\begin{array}{cccc}\text { Controls I-died August } & 8 . \\ \text { “ } & 2-“ & \text { " } & 12 . \\ \text { “ } & 3-“ & \text { “ } & 15 .\end{array}$

Experimental rabbits-Tuberculin and Tuberculous rabbit serum. I-died August 12.

2 - " 22.

Experimental rabbits-Tuberculous rabbit serum and insoluble residue. I-died from infected bite August 1 .

2-" August Io.

In order to test the properties of the products obtained from the treatment of the tubercle bacilli according to the directions of Dr. Vaughan the following tests were made.

Some of the soluble portion injected subcutaneously into a guinea pig produced the following symptoms: nervousness, chilliness, weakness in the hind quarters, convulsions and death in about 45 minutes.

Injections of the alcoholic insoluble portion did not cause symptoms of any sort.

A small quantity of this insoluble portion was injected into each of three guinea pigs. After three weeks two of these pigs were injected intraperitoneally with an emulsion of tubercle bacilli, from which the split products had been prepared, with the following results.

Pig No. I. After 30 minutes, restlessness followed by weakness in the hind legs; violent convulsions followed by extreme fatigue. These convulsions continued during the day and the pig died finally in a convulsion 6 hours after the injection.

No. 2 had lost a great deal in weight and was undoubtedly tuberculous. 
The autopsy showed undissolved bodies of tubercle bacilli spread over the omentum.

Pig No. 2. Some restlessness but no other sign that it was affected by the injection. Two days later this pig was seized with a convulsion and died.

The autopsy showed undissolved bodies of tubercle bacilli not spread over the omentum but collected together in small groups. The edge of the spleen was covered with small abscesses which did not show tubercle bacilli.

The serum of rabbits treated according to experiment two (2) acquired a clumping property for the tubercle bacilli. This was shown by each of the two rabbits that are now duplicating the successful experiment No. 2.

An emulsion of bovine tuberculosis was mixed with clear serum drawn from these animals six days after the last injection.

A mixture of this serum from each rabbit and a uniform suspension of tubercle bacilli after standing overnight at room temperature was found to cause well defined clumping. The control in normal sodium chloride solution was not clumped.

A knowledge of substances which counteract the toxic effects of the infecting organism is of great value in attempts at immunization.

The fact that "tuberculin" the toxic extract of the tubercle bacillus is an arterial blood depressor immediately suggests that the extract of the suprarenal gland might antagonize it. We have carried out experiments of this nature and find that according to the amount of tuberculin and suprarenal extract in the mixture one can produce a depression or rise at will.

The depressor substance in tuberculin then can be measured by suprarenal extract of known strength.

If the depressor substance in tuberculin is a necessary constituent for tuberculin as a diagnostic and therapeutic agent, then we have an easy and simple method of measuring its strength.

We have injected tuberculin into dogs from which we have removed one and both suprarenals. The results bear out the theory, but have brought to light so much that is new and interesting that we will reserve the discussion for a future paper. 